\title{
Rare and Semi-rare Decays of Beauty Mesons in ATLAS
}

Wolfgang Walkowiak*, on behalf of the ATLAS Collaboration

University of Siegen, 57068 Siegen, Germany

E-mail: walkowiak@hep.physik.uni-siegen.de

The ATLAS experiment at the Large Hadron Collider has performed measurements of the rare flavor-changing neutral-current processes $b \rightarrow s \mu^{+} \mu^{-}$and $B_{(s)}^{0} \rightarrow \mu^{+} \mu^{-}$which are sensitive to New Physics effects. This contribution presents recent ATLAS results from the angular analysis of the $B_{d}^{0} \rightarrow K^{* 0} \mu^{+} \mu^{-}$decay with LHC Run 1 data, the $\mathscr{B}\left(B_{(s)}^{0} \rightarrow \mu^{+} \mu^{-}\right)$measurement with 2015 and 2016 data as well as projections for the $B_{d}^{0} \rightarrow K^{* 0} \mu^{+} \mu^{-}$and $\mathscr{B}\left(B_{(s)}^{0} \rightarrow \mu^{+} \mu^{-}\right)$measurements for the High-Luminosity LHC phase.

18th International Conference on B-Physics at Frontier Machines - Beauty2019 -

29 September / 4 October, 2019

Ljubljana, Slovenia

${ }^{*}$ Speaker. 


\section{Introduction}

New physics beyond the Standard Model (SM) may manifest itself in angular distributions of $b \rightarrow s \mu^{+} \mu^{-}$processes or the branching fractions of very rare $B$ meson decays. The ATLAS experiment [1] at the Large Hadron Collider (LHC) [2] at CERN performs indirect searches for New Physics by an angular analysis of the $B_{d}^{0} \rightarrow K^{* 0} \mu^{+} \mu^{-}$decay and measuring the branching fractions of the rare decays $B_{s}^{0} \rightarrow \mu^{+} \mu^{-}$and $B^{0} \rightarrow \mu^{+} \mu^{-}$. In addition, expected sensitivities for the angular analysis in the decay channel $B_{d}^{0} \rightarrow K^{* 0} \mu^{+} \mu^{-}$and for the branching fractions of the rare decays $B_{(s)}^{0} \rightarrow \mu^{+} \mu^{-}$at the High-Luminosity LHC (HL-LHC) [3] are presented.

\section{Angular Analysis of $B_{d}^{0} \rightarrow K^{* 0} \mu^{+} \mu^{-}$}

Mediated by flavour-changing neutral curents (FCNC), in the Standard Model (SM) the decay $B_{d}^{0} \rightarrow K^{* 0} \mu^{+} \mu^{-}$with $K^{* 0} \rightarrow K^{+} \pi^{-}$proceeds via loop diagrams. The complex angular structure of this decay is fully described by three angles and the dimuon invariant mass squared $q^{2}$. Multiple angular observables provided by this decay are sensitive to different types of New Physics (NP). A $3.4 \sigma$ deviation from SM calculations is reported by the LHCb collaboration [4]. The measurement [5] with the ATLAS detector [1], using $20.3 \mathrm{fb}^{-1}$ of $p p$ collision data at a centreof-mass energy of $\sqrt{s}=8 \mathrm{TeV}$ collected in 2012, adopts the LHCb analysis method including the definitions of angular observables and of optimised parameters $P_{i}^{(/)}$[6]. The latter are designed to minimise uncertainties from hadronic form factors and therefore increase the sensitivity to NP. Due to the limited statistics a set of trigonometric transformations of the angular variables [6] is employed in the analysis presented.

In order to maximise the signal yield, data are combined from trigger chains with one, two or at least three identified muons. Furthermore this ensures a sensitivity of the analysis down to the kinematic threshold of $q^{2}=0.04 \mathrm{GeV}^{2}$. Signal candidates are reconstructed from two charged tracks satisfying $m_{K \pi} \in[846,946] \mathrm{MeV}$ and two muons, requiring $m_{K \pi \mu \mu} \in[5110,5700] \mathrm{MeV}$. Cutbased selections on the vertex fit quality $\chi^{2} /$ n.d.f. $<2$, the $B^{0}$ lifetime significance $t / \sigma_{t}>12.75$, the pointing angle $\cos \theta>0.999$ and the $K^{\star 0}$ momentum $p_{\mathrm{T}}\left(K^{\star 0}\right)>3 \mathrm{GeV}$ are used to suppress the combinatorial background. This selection results in a data sample of 787 events in the signal range of $q^{2} \in[0.04,6.0] \mathrm{GeV}^{2}$. Data with a $q^{2}$ above $6 \mathrm{GeV}^{2}$ are excluded in order to suppress a radiative tail from $B^{0} \rightarrow K^{\star 0} J / \psi$ events.

To extract the angular parameters an extended unbinned maximum-likelihood fit to the invariant mass $m_{K \pi \mu \mu}$ and the angular distributions $\cos \theta_{K}, \cos \theta_{L}$ and $\phi$ is performed in six bins of $q^{2}$, with three of the bins overlapping. The fit yields a total of $342 \pm 39$ signal events. A distinct background contribution from $B^{0} \rightarrow D^{0} / D_{(s)}^{+} X$ decays at $\cos \theta_{L} \sim 0.7$ is excluded by vetoing the $D^{0} / D_{(s)}^{+}$mass ranges. The background from fake $K^{\star 0}$ candidates and $B^{+} \rightarrow K^{+} / \pi^{+} \mu^{+} \mu^{-}$decays, observed at $\cos \theta_{K} \sim 1$, is treated as a systematic uncertainty with the fake $K^{\star 0}$ candidates providing the largest contribution. Overall, the measurement is largely dominated by statistical uncertainty.

Figure 1 compares the results for the $P_{4}^{\prime}$ and $P_{5}^{\prime}$ parameters to the theoretical computations of Jäger and Camalich (JC) [9, 10], Descotes-Genon et al. (DHMV) [8] and Ciuchini et al. (CFFMPSV) [7]. Experimental results from LHCb [4], CMS [11] and Belle [12] are overlaid as well. The $P_{4}^{\prime}$ and $P_{5}^{\prime}$ measurements in the $q^{2} \in[4.0,6.0] \mathrm{GeV}^{2}$ bin differ by $\sim 2.7 \sigma$ from the 

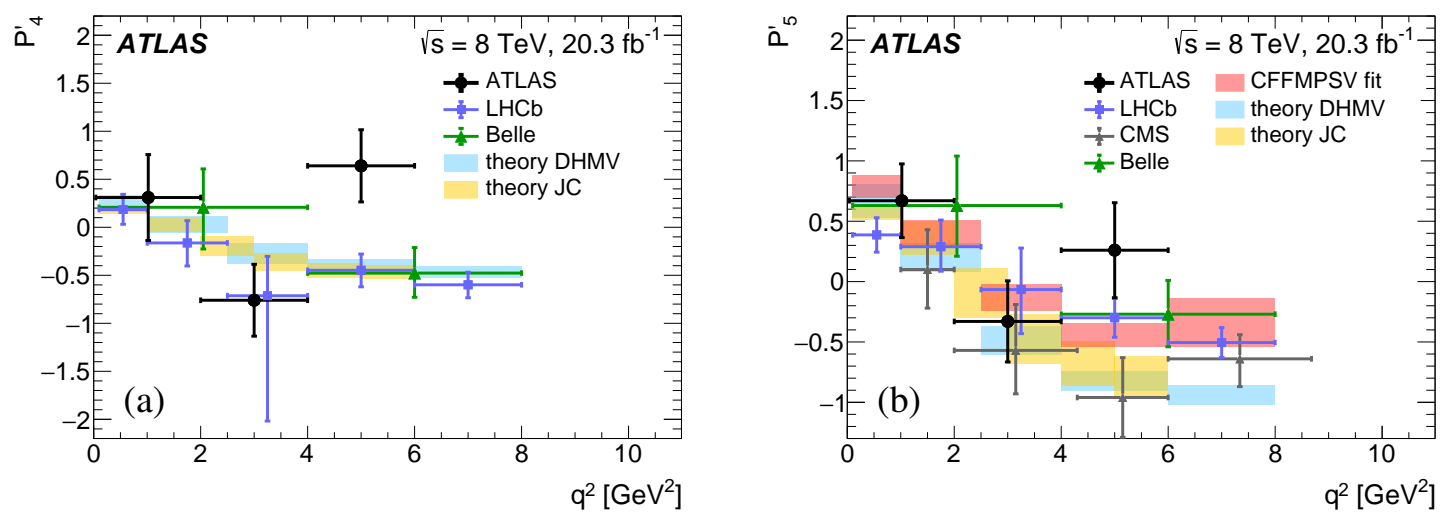

Figure 1: The measured values of $P_{4}^{\prime}$ (a) and $P_{5}^{\prime}$ (b) compared with predictions from the theoretical groups CFFMPSV [7] (only for $P_{5}^{\prime}$ ), DHMV [8] and JC [9, 10]. Experimental results from LHCb [4], CMS [11] (only for $P_{5}^{\prime}$ ) and Belle [12] are shown as well. Figures taken from [5].

DHMV model, a deviation observed similarily by the LHCb collaboration [4]. Overall, all ATLAS measurements are compatible with the different predictions at the three-standard-deviation level as well as with the results provided by the other experiments.

\section{Branching fractions of $B_{s}^{0} \rightarrow \mu^{+} \mu^{-}$and $B^{0} \rightarrow \mu^{+} \mu^{-}$}

The rare decays $B_{s}^{0} \rightarrow \mu^{+} \mu^{-}$and $B^{0} \rightarrow \mu^{+} \mu^{-}$, which are sensitive to New Physics in the decays via loop diagrams, are highly suppressed in the Standard Model (SM) with predicted branching fractions [13,14] of $(3.65 \pm 0.23) \times 10^{-9}$ and $(1.06 \pm 0.09) \times 10^{-10}$, respectively. The ATLAS Run 1 result [15] is compatible with the SM at the $2 \sigma$ level, and the $\mathscr{B}\left(B_{(s)}^{0} \rightarrow \mu^{+} \mu^{-}\right)$values are lower than the CMS-LHCb combined result [16]. Recent measurements by the LHCb [17] and CMS [18] collaborations, including part of the Run 2 data, set upper limits of $\mathscr{B}\left(B^{0} \rightarrow \mu^{+} \mu^{-}\right)<$ $3.4 \times 10^{-10}$ and $\mathscr{B}\left(B^{0} \rightarrow \mu^{+} \mu^{-}\right)<3.6 \times 10^{-10}$ at $95 \%$ confidence level (CL), respectively, which reduces the tension in this parameter.

The updated ATLAS measurement [19] of the $B_{(s)}^{0} \rightarrow \mu^{+} \mu^{-}$branching fractions includes $36.2 \mathrm{fb}^{-1}$ of data taken at a centre-of-mass energy of $13 \mathrm{TeV}$ during 2015 and 2016 (LHC Run 2) and a combination with the result based on $25 \mathrm{fb}^{-1}$ data taken at 7 and $8 \mathrm{TeV}$ during LHC Run 1. For Run 2 , events triggered by two muons $\left(p_{\mathrm{T}}\left(\mu_{1}\right)>6 \mathrm{GeV}, p_{\mathrm{T}}\left(\mu_{2}\right)>4 \mathrm{GeV},|\eta|<2.5\right)$ with the invariant di-muon mass $m_{\mu^{+} \mu^{-}}$in the range of 4 to $8.5 \mathrm{GeV}$ are selected. The dominant combinatorial background ( $b \rightarrow \mu X \times \bar{b} \rightarrow \mu X$ pairs) is rejected by a 15 -variable Boosted Decision Tree (BDT) which is trained and tested on data sidebands and simulated signal events. Tails from partially reconstructed $b \rightarrow \mu^{+} \mu^{-} X$ decays like $B \rightarrow \mu^{+} \mu^{-} X, B \rightarrow c \mu X \rightarrow s(d) \mu^{+} \mu^{-} X$ or $B_{c} \rightarrow$ $J / \psi \mu v$, which involve real di-muons at low $m_{\mu^{+} \mu^{-}}$, and semi-leptonic decays $\left(B_{(s)} / \Lambda_{b}^{0} \rightarrow h \mu v\right.$ with $h=\pi, K, p)$ contribute to the signal region and are taken into account in the signal fit. A small contribution of $B \rightarrow h h^{\prime}\left(h^{(\prime)}=\pi^{ \pm}, K^{ \pm}\right)$decays, with hadrons misidentified as muons, peaks in the $B_{(s)}^{0} \rightarrow \mu^{+} \mu^{-}$signal region contributing $2.9 \pm 2.0$ events after a "tight" muon selection is applied. The yield in the normalisation channel $B^{ \pm} \rightarrow J / \psi K^{ \pm}$with $J / \psi \rightarrow \mu^{+} \mu^{-}$is determined by an unbinned maximum likelihood fit to $m_{J / \psi K^{ \pm}}$while the efficiency relative to $B_{(s)}^{0} \rightarrow \mu^{+} \mu^{-}$is extracted 

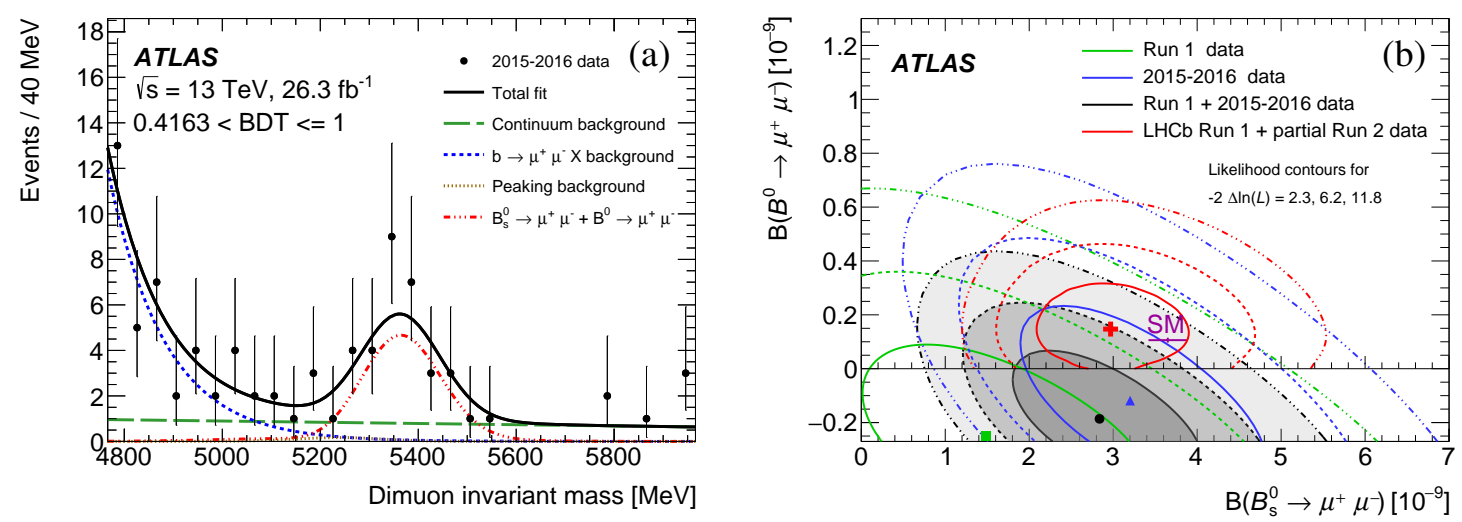

Figure 2: (a): Dimuon invariant mass distribution in the unblinded data, for the highest interval of BDT output. The result of the maximum-likelihood fit is superimposed. The total fit is shown as a continuous line, with the dashed lines corresponding to the observed signal component, the $b \rightarrow \mu^{+} \mu^{-} X$ background, and the continuum background. The signal components are grouped in one single curve, including both the $B_{s}^{0} \rightarrow \mu^{+} \mu^{-}$and the (negative) $B^{0} \rightarrow \mu^{+} \mu^{-}$component. The curve representing the peaking $B_{(s)}^{0} \rightarrow h h^{\prime}$ background lies very close to the horizontal axis [19].

(b): Likelihood contours for the combination of the Run 1 and 2015-2016 Run 2 results (shaded areas). The contours are obtained with the combination of the likelihood for the two analyses, for values of $-2 \Delta \ln \mathscr{L}$ equal to 2.3, 6.2 and 11.8. The contours for the individual 2015-2016 Run 2 and Run 1 results as well as the ones from the latest $\mathrm{LHCb}$ result [17] are overlaid. The SM predictions and their uncertainties [13] are included. Figures taken from [19].

from Monte Carlo (MC) within a fiducial volume defined by $p_{\mathrm{T}}(B)>8 \mathrm{GeV}$ and $\left|\eta_{B}\right|<2.5$. The overall efficiency ratio $R_{\varepsilon}=\varepsilon_{J / \psi K^{ \pm}} / \varepsilon_{\mu^{+} \mu^{-}}$is $0.1176 \pm 0.0009$ (stat.) \pm 0.0047 (syst.) with the largest contribution to the systematic uncertainties originating from data-MC discrepancies in the BDT input quantities. A correction of $2.7 \%$ has been applied to $R_{\varepsilon}$ to account for the effective $B_{s}^{0}$ lifetime.

Due to the limited mass resolution the overlapping $B_{s}^{0}$ and $B_{d}^{0}$ peaks are statistically separated by an unbinned maximum likelihood fit to the $m_{\mu^{+}} \mu^{-}$distributions in four BDT bins. The signal and $B \rightarrow h h^{\prime}$ distributions are modelled by three double-Gaussian PDFs, each with a common mean, while the background is described by a first-order polynomial (combinatorial background) in combination with an exponential distribution ( $b \rightarrow \mu^{+} \mu^{-} X$ and semi-leptonic background) whose shape parameters and normalisations are obtained from data (Figure 2 (a)).

For the Run 2 data, yields of $N_{s}=80 \pm 22 B_{s}^{0} \rightarrow \mu^{+} \mu^{-}$and $N_{d}=-12 \pm 20 B^{0} \rightarrow \mu^{+} \mu^{-}$events are extracted, consistent with SM expectations of $N_{s}^{\mathrm{SM}}=91$ and $N_{d}^{\mathrm{SM}}=10$, respectively. Employing a Neyman construction a branching fraction of $\mathscr{B}\left(B_{s}^{0} \rightarrow \mu^{+} \mu^{-}\right)=\left(3.21_{-0.91}^{+0.96} \text { (stat. }\right)_{-0.30}^{+0.49}$ (syst. $\left.)\right) \times$ $10^{-9}$ and an upper limit of $\mathscr{B}\left(B^{0} \rightarrow \mu^{+} \mu^{-}\right)<4.3 \times 10^{-10}$ at $95 \%$ CL are obtained. A combination of the likelihood contours of the Run 2 (2015 and 2016) and Run 1 results (Figure 2 (b)) is compatible with the SM at the $2.4 \sigma$ level and results in $\mathscr{B}\left(B_{s}^{0} \rightarrow \mu^{+} \mu^{-}\right)=\left(2.8_{-0.7}^{+0.8}\right) \times 10^{-9}$ and $\mathscr{B}\left(B^{0} \rightarrow \mu^{+} \mu^{-}\right)<2.1 \times 10^{-10}$ at $95 \%$ CL. 

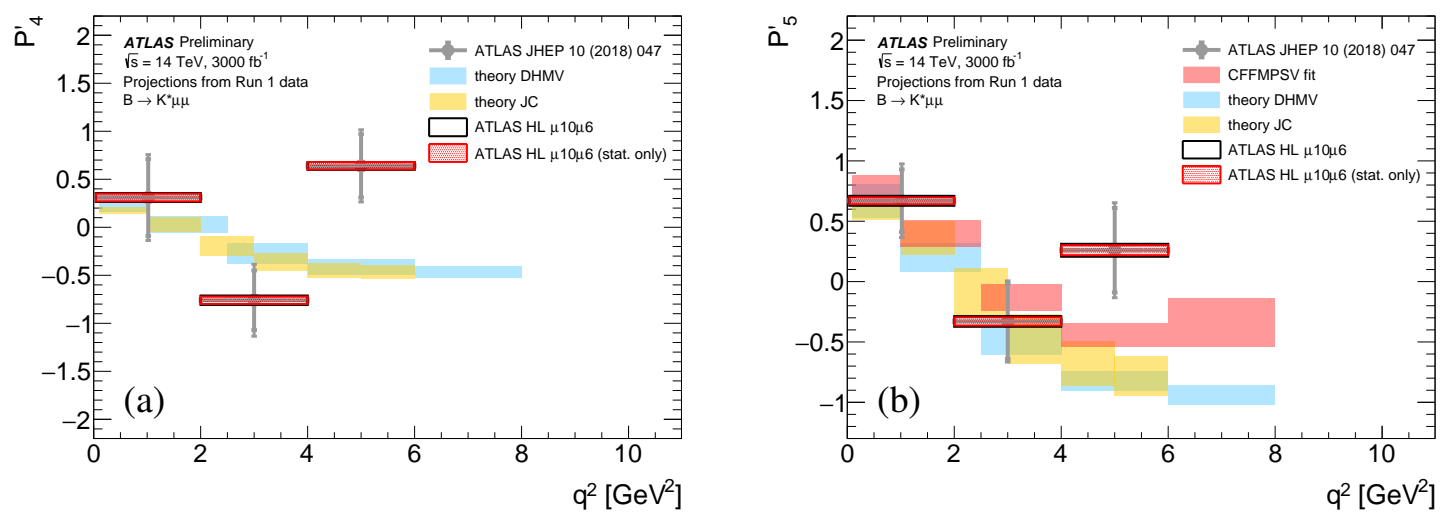

Figure 3: Projected ATLAS HL-LHC measurement precision in the $P_{4}^{\prime}$ (a) and $P_{5}^{\prime}$ (b) parameters for the intermediate $\mu 10 \mu 6$ trigger scenario compared to the ATLAS Run 1 measurement. Alongside, theory predictions (CFFMPSV [7], DHMV [8] and JC [9, 10]) are also shown. Both the projected statistical and the total (statistical and systematic) uncertainties are shown. While the HL-LHC toy-MC were generated with the DHMV central values of the $P_{4}^{\prime}$ and $P_{5}^{\prime}$ parameters, in these plots the central values are moved to the ATLAS Run 1 measurement for better visualization of the improvement in the precision [20].

\section{High-Luminosity LHC Prospects}

At the High-Luminosity LHC (HL-LHC), due to a new all-silicon Inner Tracker (ITk) the mass resolution of the 4-prong FCNC decay $B_{d}^{0} \rightarrow K^{* 0} \mu^{+} \mu^{-}$is expected to improve by $30 \%$ with respect to the Run 1 measurement which is used as a baseline for the HL-LHC projections of the measurement. For the HL-LHC case three potential trigger scenarios are considered: two muons with $p_{\mathrm{T}}>10 \mathrm{GeV}$ ("conservative"), one muon with $p_{\mathrm{T}}>10 \mathrm{GeV}$ and another with $p_{\mathrm{T}}>6 \mathrm{GeV}$ ("intermediate") as well as two muons with $p_{\mathrm{T}}>6 \mathrm{GeV}$ ("high yield") providing 50, 160 and 250 times the Run 1 statistics, respectively. This includes a factor 1.7 due to the increase of the $b$ production cross-section as the center-of-mass energy of the $p p$ collisions rises from $8 \mathrm{TeV}$ to $14 \mathrm{TeV}$. Estimates for the achievable experimental precision are obtained from pseudo-MC experiments based on the Run 1 signal and background angular distributions and by applying the same fitting procedure as in the Run 1 analysis.

Assuming that the increased statistics will allow for an improved fit model and a better understanding of the exclusive backgrounds, the corresponding systematic uncertainties are scaled by $1 / \sqrt{L_{\text {int }}}$. The expected improvement in the measurement accuracy of the $P_{4}^{\prime}$ and $P_{5}^{\prime}$ parameters is demonstrated in Figure 3, compared to the current theoretical predications. Depending on the trigger scenario, the measurement precision for the $P_{5}^{\prime}$ parameter is expected to improve by a factor 5,8 or 9 relative to the Run 1 measurement.

The branching fraction measurement of the very rare decays $B_{s}^{0} \rightarrow \mu^{+} \mu^{-}$and $B^{0} \rightarrow \mu^{+} \mu^{-}$will also benefit from the increased statistics and the improved invariant mass resolution at the HL-LHC. The separation of the $B_{s}^{0}$ and $B_{d}^{0}$ mass peaks increases by a factor of $1.65(1.5)$ to $2.3 \sigma(1.3 \sigma)$ in the barrel (end-cap) region compared to Run 1 [21].

The projection of the ATLAS detector performance for measuring $\mathscr{B}\left(B_{(s)}^{0} \rightarrow \mu^{+} \mu^{-}\right)$with the expected datasets during the full LHC Run $2\left(130 \mathrm{fb}^{-1}\right)$ and at the HL-LHC $\left(3000 \mathrm{fb}^{-1}\right)$ [22] us- 

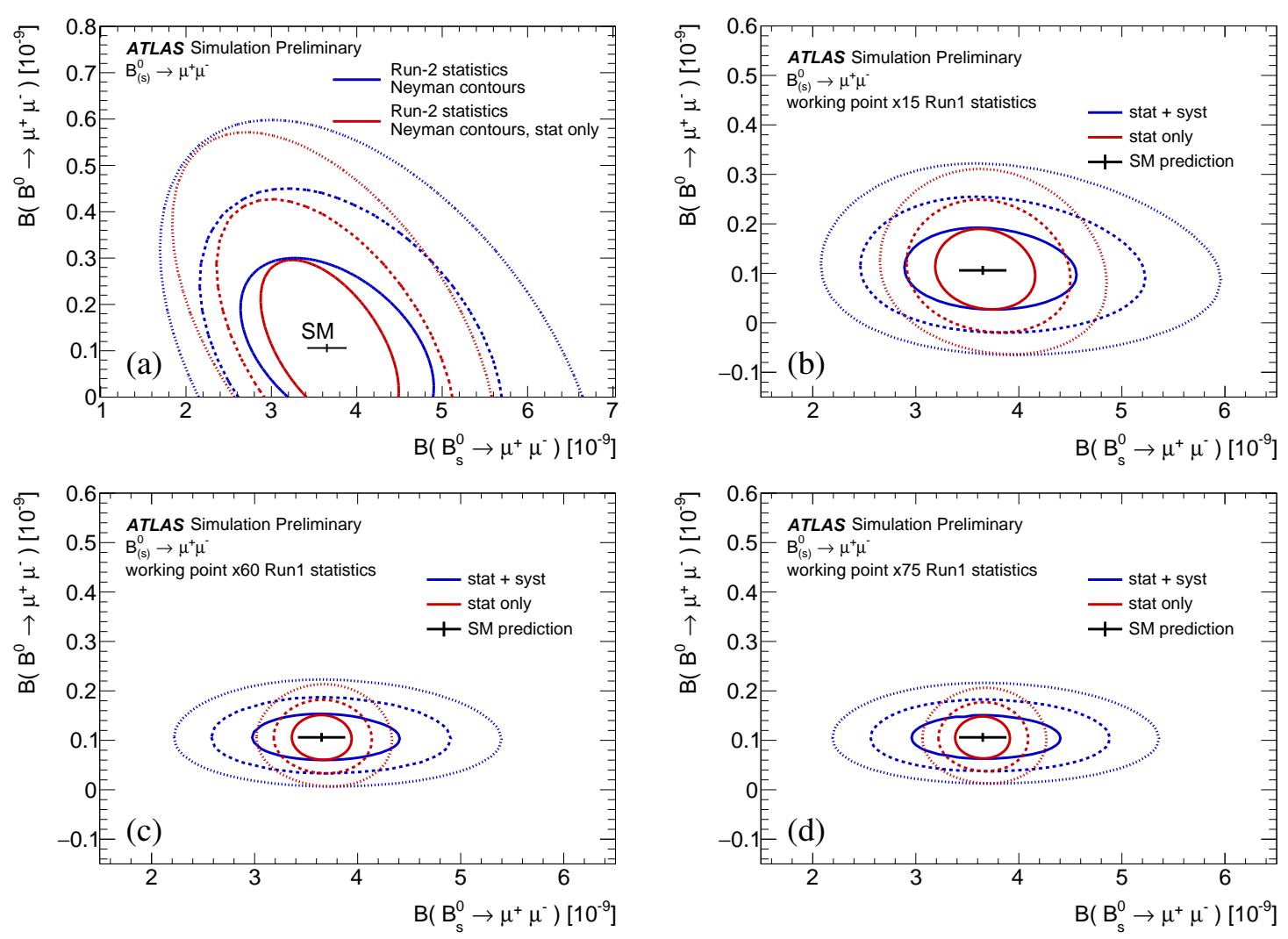

Figure 4: (a): Comparison of $68.3 \%$ (solid), $95.5 \%$ (dashed) and $99.7 \%$ (dotted) confidence level contours obtained exploiting the 2D Neyman belt construction for the full LHC Run 2 case [22]. Red contours are statistical only; blue contours include systematics uncertainties from the ATLAS Run 1 analysis [15] extrapolated to Run 2 statistics. The black points show the SM theoretical prediction and its uncertainty [13]. (b) - (d): Comparison of confidence level profiled likelihood ratio contours for (b) the "conservative", (c) the "intermediate" and (d) the "high-yield" HL-LHC extrapolation with $\times 15, \times 60$ and $\times 75$ the Run 1 statistics for the $(10 \mathrm{GeV}, 10 \mathrm{GeV})$, the $(6 \mathrm{GeV}, 10 \mathrm{GeV})$ and the $(6 \mathrm{GeV}, 6 \mathrm{GeV})$ dimuon trigger scenarios, respectively [22].

ing pseudo-MC experiments is based on the likelihood of the Run 1 analysis. The signal statistics estimate for the Run 2 scenario applies scaling factors for the integrated luminosity, the crosssection increase due to the higher center-of-mass energy of $13 \mathrm{TeV}$ and the muon pair selection with topological triggers with $\left(p_{\mathrm{T}}\left(\mu_{1,2}\right)>6 \mathrm{GeV}\right)$ or $\left(p_{\mathrm{T}}\left(\mu_{1}\right)>6 \mathrm{GeV}, p_{\mathrm{T}}\left(\mu_{2}\right)>4 \mathrm{GeV}\right)$ thresholds resulting in 7 times the number of signal events in Run 1. The contours of the 2-dimensional Neyman construction (Figure 4 (a)) include the external systematic uncertainties on the $b$-quark fragmentation fractions $f_{s} / f_{d}$ and $\mathscr{B}\left(B^{ \pm} \rightarrow J / \psi K^{ \pm}\right)$which were kept the same as in the Run 1 analysis as well as internal ones like the fit shapes and efficiencies which were scaled according to the increase in statistics. For the HL-LHC case the same three potential trigger scenarios as for the $B_{d}^{0} \rightarrow K^{* 0} \mu^{+} \mu^{-}$analysis are considered resulting in 15 ("conservative"), 60 ("intermediate") and 75 ("high yield") times the Run 1 statistics, respectively. The profile likelihood contours of pseudo-experiments based again on the likelihood of the Run 1 analysis demonstrate the increased sensitivity of the ATLAS detector for $\mathscr{B}\left(B_{s}^{0} \rightarrow \mu^{+} \mu^{-}\right)$and $\mathscr{B}\left(B^{0} \rightarrow \mu^{+} \mu^{-}\right)$at the HL-LHC (Fig- 
ure 4 (b) - (d)). The uncertainty on the $f_{s} / f_{d}$ value, conservatively taken as $8.3 \%$ from the ATLAS measurement [23], dominates the systematic uncertainty contributions on $\mathscr{B}\left(B_{s}^{0} \rightarrow \mu^{+} \mu^{-}\right)$.

\section{Summary}

Measurements of semi-rare flavor-changing neutral-current decays and of very rare decays, both sensitive to New Physics, by the ATLAS collaboration at the LHC have been presented.

The results of the angular analysis of the $B_{d}^{0} \rightarrow K^{* 0} \mu^{+} \mu^{-}$decay with $20.3 \mathrm{fb}^{-1}$ of Run 1 data agree well with the theoretical predictions in the SM and other measurements, with the largest deviation from theory $(\sim 2.7 \sigma)$ observed for the $P_{4}^{\prime}$ and $P_{5}^{\prime}$ parameters in the $q^{2} \in[4.0,6.0] \mathrm{GeV}^{2}$ bin.

The results for $\mathscr{B}\left(B_{s}^{0} \rightarrow \mu^{+} \mu^{-}\right)$and the search for the decay $\mathscr{B}\left(B^{0} \rightarrow \mu^{+} \mu^{-}\right)$with $36.2 \mathrm{fb}^{-1}$ of Run 2 data agree with the Standard Model and other measurements. There is no sign for the decay $B^{0} \rightarrow \mu^{+} \mu^{-}$in ATLAS data, but ATLAS will add the data taken in 2017 and 2018 to the analysis $\left(\sim 107 \mathrm{fb}^{-1}\right)$.

Both analyses will profit considerably from the increased statistics expected from the $3000 \mathrm{fb}^{-1}$ of HL-LHC data as well as detector improvements providing better mass and proper decay time resolutions. This will allow more stringent tests of the Standard Model.

\section{Acknowledgments}

This work was partially supported by grants of the German Federal Ministry of Education and Research (BMBF) and the German Helmholtz Alliance "Physics at the Terascale".

\section{References}

[1] ATLAS Collaboration, The ATLAS Experiment at the CERN Large Hadron Collider, 2008 JINST 3 S08003

[2] L. Evans and P. Bryant (editors), LHC Machine, 2008 JINST 3 S08001

[3] G. Apollinari, I. Béjar Alonso, O. Brüning, P. Fessia, M. Lamont, L. Rossi, L. Tavian (editors), High-Luminosity Large Hadron Collider (HL-LHC), Technical Design Report V. 0.1, CERN Yellow Reports Vol. 4/2017, CERN-2017-007-M (CERN, Geneva, 2017) [https://cds.cern.ch/record/2284929]

[4] LHCb Collaboration, Angular analysis of the $B_{d}^{0} \rightarrow K^{* 0} \mu^{+} \mu^{-}$decay using $3 \mathrm{fb}^{-1}$ of integrated luminosity, JHEP 02 (2016) 104 [arXiv: 1512.04442 ]

[5] ATLAS Collaboration, Angular analysis of the $B_{d}^{0} \rightarrow K^{* 0} \mu^{+} \mu^{-}$decay using $3 \mathrm{fb}^{-1}$ of integrated luminsity, JHEP 10 (2018) 047 [arXiv: 1805.04000 ]

[6] LHCb Collaboration, Measurement of Form-Factor Independent Observables in the Decay $B_{d}^{0} \rightarrow K^{* 0} \mu^{+} \mu^{-}$, Phys. Rev. Lett. 111 (2013) 191801 [arXiv:1308.1707]

[7] M. Ciuchini et al., $B \rightarrow K^{\star} \ell^{+} \ell^{-}$decays at large recoil in the Standard Model: a theoretical reappraisal, JHEP 06 (2016) 116, [arXiv: 1512 .07157]

[8] S. Descotes-Genon et al., On the impact of power corrections in the prediction of $B \rightarrow K^{\star} \mu+\mu-$ observables, JHEP 12 (2014) 125, [arXiv: 1407 . 8526] 
[9] S. Jäger and J. Martin Camalich, On $B \rightarrow V \ell^{+} \ell^{-}$at small dilepton invariant mass, power corrections and new physics, JHEP 05 (2013) 043 [arXiv: 1212.2263]

[10] S. Jäger and J. Martin Camalich, Reassessing the discovery potential of the $B \rightarrow K^{\star} \ell^{+} \ell^{-}$decays in the large-recoil region: SM challenges and BSM opportunities, Phys. Rev. D 93 (2016) 014028 [arXiv:1412.3183]

[11] CMS Collaboration, Measurement of angular parameters from the decay $B_{d}^{0} \rightarrow K^{* 0} \mu^{+} \mu^{-}$in proton-proton collisions at $\sqrt{s}=8 \mathrm{TeV}$, Phys. Lett. B 781 (2018) 517 [arXiv: 1710.02846 ]

[12] Belle Collaboration, Angular analysis of $B^{0} \rightarrow K^{\star}(892)^{0} \ell^{+} \ell^{-}$, BELLE-CONF-1603 [arXiv:1604.04042]

[13] C. Bobeth et al., $B_{s, d}^{0} \rightarrow \ell^{+} \ell^{-}$in the Standard Model with Reduced Theoretical Uncertainty, Phys. Rev. Lett. 112 (2014) 101801 [arXiv: 1311.0903 ]

[14] M. Beneke, C. Bobeth and R. Szafron, Power-enhanced leading-logarithmic QED corrections to $B_{q} \rightarrow \mu^{+} \mu^{-}$, JHEP 10 (2019) 232 [arXiv:1908.07011]

[15] ATLAS Collaboration, Study of the rare decays of $B_{s}^{0}$ and $B_{d}^{0}$ into muon pairs from data collected during the LHC Run 1 with the ATLAS detector, Eur. Phys. J. C (2016) 76:513 [arXiv:1604.04263]

[16] CMS and LHCb Collaborations, Observation of the rare $B_{s}^{0} \rightarrow \mu^{+} \mu^{-}$decay from the combined analysis of CMS and LHCb data, Nature, 522 68-72, 2015 [arXiv: 1411.4413 ]

[17] LHCb Collaboration, Measurement of the $B_{s}^{0} \rightarrow \mu^{+} \mu^{-}$Branching Fraction and Effective Lifetime and Search for $B^{0} \rightarrow \mu^{+} \mu^{-}$Decays, Phys. Rev. Lett. 118 (2017) 191801 [arXiv:1703.05747]

[18] CMS Collaboration, Measurement of properties of $B_{s}^{0} \rightarrow \mu^{+} \mu^{-}$decays and search for $B^{0} \rightarrow \mu^{+} \mu^{-}$with the CMS experiment, submitted to JHEP, CMS-PAS-BPHY-16-004 [arXiv:1910.12127]

[19] ATLAS Collaboration, Study of the rare decays of $B_{s}^{0}$ and $B_{d}^{0}$ mesons into muon pairs using data collected during 2015 and 2016 with the ATLAS detector, JHEP 04 (2019) 098 [arXiv:1812.03017]

[20] ATLAS Collaboration, $B_{d}^{0} \rightarrow K^{* 0} \mu^{+} \mu^{-}$angular analysis prospects with the ATLAS detector at the HL-LHC, ATL-PHYS-PUB-2019-003, [https : / / cds . cern. ch/record/2654519]

[21] ATLAS Collaboration, Expected peformance for an upgraded ATLAS detector at High-Luminosity LHC, ATL-PHYS-PUB-2016-026 [https : / / cds . cern. ch/record/2223839]

[22] ATLAS Collaboration, Prospects for the $\mathscr{B}\left(B_{(s)}^{0} \rightarrow \mu^{+} \mu^{-}\right)$measurements with the ATLAS detector in Run 2 LHC and HL-LHC data campaigns, ATL-PHYS-PUB-2018-005, [https://cds.cern.ch/record/2317211]

[23] ATLAS Collaboration, Determination of the ratio of b-quark fragmentation fractions $f_{s} / f_{d}$ in $p p$ collisions at $\sqrt{s}=7 \mathrm{TeV}$ with the ATLAS detector, Phys. Rev. Lett. 115, 262001 (2015) [arXiv:1507.08925] 